\title{
TURISMO RELIGIOSO Y DESTINOS MADUROS. LA GESTIÓN TURÍSTICA DE DOS CATEDRALES: PALMA DE MALLORCA Y BARCELONA
}

\author{
María Abril Sellarés \\ Universitat Autònoma de Barcelona \\ maria.abril@uab.cat $^{1}$ \\ María del Carmen Azpelicueta Criado \\ Universitat de les Illes Balears \\ m.azpelicueta@uib.eu ${ }^{2}$ \\ María Dolores Sánchez Fernández \\ Universidade A Coruña \\ msanchezf@udc.es $^{3}$
}

(Fecha envío: 02/03/15 - Fecha aceptación: 01/04/15)

\section{Resumen}

En los destinos maduros hay que buscar nuevas propuestas para poder trazar nuevas metas y ofrecer versiones innovadoras en la gestión de productos y servicios, dado que normalmente tienen servicios muy consolidados por los que atraen grandes flujos turísticos. Los destinos turísticos tratan, básicamente, de satisfacer la exigente y variada demanda turística, con un reto añadido: diversificar los productos y aumentar el gasto medio del turista así como disminuir la estacionalidad (IDOM, 2011). En base a estas realidades surge el interés de este estudio por conocer en profundidad un Recurso Patrimonial tratado como Recurso Turístico en dos destinos maduros del Mediterráneo español: Las Catedrales de Palma de Mallorca y de Barcelona, que aún en la distancia, tienen una serie de elementos y patrones en común. Dos ciudades, Palma de Mallorca y Barcelona que atraen por una oferta de servicios turísticos donde sus Catedrales están consideradas de rango 2 según la clasificación de CICATUR-OEA.

\section{Palabras clave}

Barcelona, Mallorca, catedral, destino, producto, recurso patrimonial, turista

\begin{abstract}
In consolidate destinations it's necessary to look for new proposals to set new goals and deliver innovative versions management products and services, because they usually have well established services which attract large number of tourists. Tourist destinations trying to satisfy the demanding and increasing more varied tourist demand, an added new challenges: The diversification of products, the increase of the average expense of the tourist and the reduction of the seasonal nature (IDOM, 2011). Based on these realities there arises the interest of this study to know in depth an Heritage Resource treated as a Tourist Resources, belonging to two consolidate destinations of Mediterranean places: The Cathedrals of Palma de Mallorca and Barcelona, still in the distance, there has a series of elements and patterns together. Two cities, Palma de Mallorca and Barcelona that attract by offering diverse tourists services, where the Cathedrals are considered Rank 2 according to the classification by CICATUR-OEA.
\end{abstract}

\section{Keywords}

Barcelona, Majorca, cathedral, destination, product, heritage resource, tourist

\footnotetext{
${ }^{1}$ Profesora de Patrimonio Cultural en la EUTDH-UAB. Licenciada en Humanidades, Máster en Gestión Cultural. Doctoranda Ciencias Sociales, Didáctica del Patrimonio.

2 Licenciada en Ciencias del Trabajo. Doctoranda Economía Empresa. Programa FPU M‥ de Educación, Cultura y Deportes.

${ }^{3}$ Profesora en el área de Organización de Empresas. Doctora en Competitividad, Innovación y Desenvolvimiento: Análisis económico y empresarial.
} 
Creatori serviunt omnia subjecta, sub mensura, numero, pondere perfecta.

Ad invisibilia, per haec intellecta,

sursum trahit hominem ratio directa

'Poèmes de Gautier de Châtillon' WILMART, 1937

\section{INTRODUCCIÓN}

Si el concepto Turismo acoge un profundo y mágico contenido y sentido (Barbosa, 2007), el término Catedral introduce un mundo de significados - significantes y, alude, en su génesis, a una realidad arquitectónica muy específica la iglesia de una diócesis eclesiástica, la sede del Obispo.

En el ámbito turístico mientras las motivaciones van inexorablemente unidas a la demanda turística, los recursos forman parte de la oferta que al aglutinar una serie de productos se ponen a disposición del turista (Blasco Peris, 2006). Los núcleos que forman ese producto se resumen en tres: Empresas, Infraestructuras y Recursos.

Los servicios turísticos son los bienes y servicios ofrecidos por las empresas del sector, que van a satisfacer las necesidades, actuales o futuras, de los turistas. Y finalmente, las infraestructuras son aquellos elementos físicos localizados en un destino, que pueden ser públicos o privados necesarios para acceder al lugar y disfrutar de la oferta turística. (Pulido Fernández, Sáez Cala, Antonia, 2011).

Montaner define los recursos turísticos como "Aquellos que se basan en potenciales geográficos, históricomonumentales y culturales que posee un núcleo turístico como pueden ser sus playas, monumentos, paisaje, folclore, gastronomía...y que tienen un poder de atracción y motivación sobre los turistas." (Montaner Montejano, 1991:22) En base a esta definición podemos establecer tres grupos de recursos turísticos relacionados con:

1. La historia: los relacionados directamente con restos históricos y patrimonio cultural como catedrales, museos, monumentos, conjuntos arqueológicos, ciudades monumentales, etc. y los directamente relacionados con la memoria histórica.

2. La cultura viva: compuesta por las diferentes manifestaciones propias de un modelo cultural como la gastronomía, el folclore, las tradiciones, etc.

3. La naturaleza: aquellos relacionados con el agua como ríos, mares, o relativos a la tierra y sus ecosistemas como paisajes, la flora, la fauna, etc.

Es en el grupo histórico donde encuadramos la Catedral. Una de las construcciones más icónicas de la Edad Media y que ha tenido continuidad hasta nuestros días. Se convierte en una expresión cultural de primera magnitud, a la que se la dota de diversos valores: político, simbólico o estilístico y a su vez cumple una función utilitaria. Forma parte del paisaje urbano y del carácter humano, siendo voz muda de un pasado, bien patrimonial de un presente y de un futuro protegido para que las generaciones futuras sigan descubriendo tanto su estructura como sus secretos. Pero al mismo tiempo se transforma en un recurso turístico representativo de la ciudad por el que se cobra un precio de entrada al edificio, que se confronta con la noción de acceso y disfrute de monumentos significativos por parte de la ciudadanía.

El estudio de investigación sobre las Catedrales de Barcelona y Mallorca se sustenta en una serie de elementos y patrones comunes: Ambos recursos se ubican geográficamente en el Arco Mediterráneo y están situados en el centro histórico de las ciudades-capital de Barcelona y de Palma de Mallorca; históricamente se encuadrarían bajo el amparo de la familia reinante en la Corona de Aragón; artísticamente forman parte de la misma tendencia, el arte gótico y con los artistas coincidentes, a lo largo de su construcción; su función ha sido religiosa y sus organismos gestores y propietarios, las Diócesis Católicas de dichas ciudades. Por último, son un patrimonio visitable, por lo que pueden ser consideradas un Recurso Turístico enmarcado en el denominado Turismo Cultural.

Para cumplir con los objetivos del trabajo se optó por una metodología consistente en la búsqueda, lectura y análisis sistematizado de múltiples y variadas publicaciones (artículos, comunicaciones, revistas, ...) y libros de una amplia gama, que versaban sobre los dos ejes centrales del trabajo: por un lado las Catedrales y la comparativa entre la de la ciudad de Barcelona y la de Mallorca como construcciones históricas. Y por otro lado su gestión e importancia como recurso, para lo cual nos apoyamos en la elaboración de un cuestionario que nos ha permitido esclarecer y dilucidar aquellas ideas esenciales que apoyan el valor turístico de dichos recursos históricos.

\section{LA CATEDRAL: ENTRE EL TURISMO CULTURAL Y EL TURISMO RELIGIOSO}

El concepto de turismo cultural ha ido evolucionando a lo largo del tiempo. Comienza siendo una modalidad de turismo cuyo objeto es, exclusivamente, el descubrimiento de sitios y monumentos, y se convierte con el tiempo en una forma de viajar motivada por el deseo de conocer, comprender o estudiar otras culturas (IDOM, 2011). 
Las actividades que a través de ella se pueden realizar pueden ir desde visitar una tribu en el norte de Tailandia hasta visitar una Catedral en España.

En realidad, el turismo cultural se muestra como una de las tipologías turísticas más antiguas, dado que en la segunda mitad del siglo XVIII el Grand Tour mostraba como uno de sus principales atractivos el pasado creativo, cultural y político. (Marrero Rodríguez, 2009).

El concepto de turismo religioso, a juicio de la $\mathrm{OMT}^{4}$, se encuentra en los vocablos "otros motivos" que utiliza para la definición del fenómeno turístico, "el turismo comprende las actividades que realizan las personas durante sus viajes y estancias en lugares distintos al de su entorno habitual, por un período de tiempo consecutivo inferior a un año, con fines de ocio, por negocios y otros motivos" (Organización Mundial de Turismo, 1995). Concretamente, en la clasificación que la propia OMT realiza de los "otros motivos" el quinto de ellos es la religión y peregrinaciones.

A juicio de este organismo internacional, cabe inferir que, si el motivo para viajar cumple una serie de requisitos y la razón es religiosa y peregrina, será adecuado hablar de turismo religioso.

El concepto de turismo religioso podría estar estrechamente vinculado al de turismo cultural, puesto que posee los mismos elementos de oferta y demanda, aunque puede no compartir la motivación, así la diferencia estriba en que mientras en uno se comercializa la cultura, en éste se comercializa la religión y la devoción popular. El caso que nos ocupa puede complementar un nexo en común entre patrimonio religioso, cultura y turismo, eso dependerá de la manifestación que se produzca de manera puntual y de la demanda turística que tengamos en un momento determinado, convirtiendo el destino en foco de atracción turística de gran importancia (IDOM, 2011). El documento de perspectivas del turismo para el 2020 (Ministerio, 2008) apunta que el turismo religioso es uno de los segmentos emergentes en España.

\section{LA CATEDRAL: INTRODUCCIÓN HISTÓRICA. VALOR Y SIGNIFICADO}

El vocablo "Catedral" ha tenido una evolución considerable desde el origen del cristianismo. Proveniente de la palabra griega i posteriormente latinizada cathedra, designaba la silla del Obispo, ubicada tradicionalmente, en el ábside, enfrente al altar mayor y los fieles. En otras sociedades, lo que define la construcción es el término domus, latino, entendido como la casa de Dios, (italiano, Duomo, en alemán, Dom). La diferencia no es sólo semántica sino de significado, para unas culturas era la silla y metafóricamente la casa del Obispo o arzobispo y para otras culturas era propiamente la casa de Dios. El término cathedra se adjetivó al asociarlo a la ecclesia o comunidad de fieles, pasando, a lo largo del tiempo a definir el conjunto arquitectónico, antes de sustantivarse en cathedralis. Finalmente, tanto el término, Catedral, como Duomo - Dom tendrán un mismo significado la casa del Señor (Le Goff \& Schmitt, 2003).

La evolución demográfica, en el marco europeo y durante la época feudal, eclosionó a finales del siglo XII desarrollándose durante todo del siglo XIII y en algunos países a hasta mediados del siglo XIV. Las ciudades se convirtieron en el eje político, económico y social de la Europa Occidental, dejando en un segundo plano al mundo rural, los castillos y los monasterios. Si entre los siglos IX al XII la construcción eclesiástica por excelencia fue el Monasterio, a finales del siglo XII hasta bien entrado el siglo XVI la construcción fue la Catedral, que se adaptaría a nivel constructivo a las nuevas exigencias y a nivel simbólico e icónico, siendo uno de los principios base, "Dios es Luz". La construcción adaptó sus espacios a las nuevas exigencias de la liturgia, donde las fachadas quedaron bien definidas, y los programas iconográficos, adaptándose a las nuevas tendencias y espacios, siguieron manteniendo su carácter didáctico - pedagógico (Le Goff \& Schmitt, 2003).

La catedral gótica se originó, pues, en la experiencia religiosa y la especulación metafísica, en las realidades políticas e incluso materiales de la Francia del siglo XII y en el genio de los que la crearon (Von Simson, 1956). Una vez creado, el gótico se convirtió en el "idioma" conservador de la arquitectura cristiana en todo el mundo occidental desde los siglos XII al XVI, entendiendo los edificios como un todo organizado, cuyas partes adoptan formas determinadas, según su función en el conjunto (Kinder y Hilgemann, 1990) y, que si hubiera en cualquiera de ellas la más pequeña alteración, se destruiría el conjunto, puesto que se basa en la aplicación de la sección áurea a la planta, alzado y acabados.

En su valor simbólico - icónico, tenemos que hay dos grandes pilares en los que se sustentó, por un lado en una función aritmética, y por otro, en una función mística.

En función aritmética, hablar de CATEDRAL es hablar de Arquitectura, Belleza, Culto, Desarrollo, Esplendor, Fe, Gloria, Historia, Inicio, Joya, Kaos, Lugar, Llamas, Mundo, Nobleza, Oscuridad, Paz, Revelación, Sagrado, Transformación, Única, Virtud, Yugo y Zénit bajo los auspicios de los Mártires, los Santos y la Luz Celestial de

\footnotetext{
${ }^{4}$ OMT: Organización Mundial del Turismo. Se trata de un organismo especializado de las Naciones Unidas y, representa la principal institución internacional en el campo del turismo. Constituye un foro mundial para debatir cuestiones de política turística y una fuente útil de conocimientos especializados en este campo.
} 
los Cielos purificando la Tierra (García Martos, 2012), donde 24 se compone de $2+4=6$. De ahí, que las Catedrales siempre han sido un referente para el Hombre y para entendernos, cuando uno olvida su pasado está despreciándose como Ser. Una iglesia sí es un espacio para albergar a los fieles, incluso se calcula su área en función de la población y de su crecimiento, como nos lo expone Simón García en 1681. Esta arquitectura nos muestra un lenguaje intelectual universal. Líneas y números son su esqueleto que conforma el espacio y números son sus músculos, el espesor de sus paredes, pilares, columnas o contrafuertes. Estos números son intencionados y muchas veces simbólicos

En su función ético - religiosa, la CATEDRAL es la casa de Dios, y es, ante el Todopoderoso ante quien se examinan los arquitectos, escultores, vidrieros, hacedores de retablos, pintores, y patrones. La Catedral tiene que encaminarse hacia Dios, de ahí que su construcción irá hacia Él. La Catedral será la expresión del pensamiento cristiano bajo medieval. Quiere emular la grandeza de Dios con lo que verá reducir sus muros, abrirá vanos para que la Luz, que es Dios, impregne a los fieles de sabiduría y amor Divino.

Una Catedral era y es un espacio arquitectónico que engloba continente y contenido, arte, historia, tradición y la acción continuada de obispos, decanos y canónigos, así como nobles, enriqueciendo cada día los documentos, las celebraciones, los hechos y sus obras (Bassegoda, 1995). Así que nada debe extrañarnos que esta arquitectura hable, desde un lenguaje intelectual universal que es el de los números, números que son simbólicos y parlantes, y desde un lenguaje ético - religioso que se da a través de la arquitectura en sí misma, como a través de las artes que decoran dicha construcción.

\section{LA CORONA DE ARAGÓN. EL GÓTICO COMO SÍMBOLO DE SU PODER}

A partir de mediado siglo XII y hasta el siglo XV la Corona de Aragón mostró su etapa de mayor esplendor político y comercial. A nivel artístico los ecos de dicho esplendor llegaron hasta bien entrado el siglo XV. (Ainaud de Lasarte, 1992).

Las ciudades, dentro del territorio de la Corona, mostraron el reflejo de dicho apogeo de maneras distintas: Mientras en el norte del territorio peninsular en la segunda mitad del siglo XII mostraba aún la potencia que había tenido el arte románico, en la parte sur, concretamente en los territorios conquistados a los árabes, el románico se mostraba evolutivo, incluyendo características del arte gótico que ya se estaban produciendo en Francia como el arco apuntando y la bóveda de crucería, los mejores ejemplos fueron los monasterios cistercienses y partes de la Catedral de Tarragona y de la Seu Vella de Lleida.

En el siglo XIII, Jaime I el conquistador granó nuevos territorios no sólo peninsulares como Valencia, sino también insulares como casi todas las islas Baleares. A estas tierras llegó, por un lado, una nueva concepción político-administrativa, y por el otro, el mundo mercantil - comercial y artístico. El arte gótico se convertirá en el arte de la Corona, y en la medida que se expansionaba se fue uniformando ya que sus artistas fueron viajando a lo largo y ancho del territorio dejando su interpretación artística.

Su lenguaje fue original ya que supo adaptarse al medio natural, a las tradiciones culturales del país, al influjo de las tipologías arquitectónicas que introducían las nuevas órdenes mendicantes, a una cierta estética del Císter y finalmente, a una asimilación parcial del modelo catedralicio francés. El resultado fue la adopción de unas formas y técnicas constructivas propias, tanto en la arquitectura civil, como militar, como religiosa, que alcanzaron su plenitud durante la primera mitad del siglo XIV y perduraron más que en otros lugares de la Europa Occidental.

Es bajo el reinado de Jaime II, el siglo XIV que se construyeron en la corona de Aragón, las catedrales de Barcelona, Gerona, Huesca, Manresa, Mallorca y Zaragoza. Si bien cada gran construcción catedralicia adoptaría singularidades propias, en algunos casos los elementos comunes, a lo largo de su dilatada historia, permiten un estudio comparativo, es el caso de la Catedral de Barcelona y de Mallorca.

\subsection{La Catedral de Barcelona. Breve introducción histórica}

La Catedral de Barcelona es sede del Arzobispado de Barcelona. Las primeras referencias de la Diócesis eclesiástica en dicha ciudad datan del año 343 d.C., si bien tenemos noticias anteriores de la existencia de una tradición cristiana por las historias de sus mártires (Fàbrega i Grau, 1968).

En el año 599, se reunió en la Sede el Concilio de Barcelona, y en el 877 se acogieron las reliquias de Santa Eulalia, pasando a tener una doble advocación: la Santa Cruz y Santa Eulalia (nombres con las que se conoce en la actualidad)

La catedral actual se construyó sufragada por los donativos de las cofradías y gremios medievales, a lo largo de seis siglos, cerca de la Muralla, en el ángulo norte del núcleo romano - medieval y sobre las construcciones de sus predecesoras románica y visigótica (paleocristiana) con vestigios aún visibles como la capilla de Santa Lucia, los capiteles del altar principal o el baptisterio respectivamente). 
Las obras se iniciaron en 1298 siendo Obispo Bernat Pelegrí y rey de Aragón Jaume II; las obras se dieron prácticamente por finalizadas bajo el reinado de Alfonso V de Aragón y el Obispo Francesc Climent Sapera en 1448. Y finalmente, entre 1890 y 1913 el arquitecto se encargó de finalizarla con la ayuda de los planos originales, añadiendo las dos torres laterales y anexionando una fachada neogótica. Desde 1929 es Bien de Interés Cultural y Monumento Histórico-Artístico Nacional.

\subsection{La Catedral de Mallorca. Breve introducción histórica}

La promesa hecha por Jaime I el Conquistador, durante su primer viaje a Mallorca, de dedicar una iglesia a la Virgen María en Madîna Mayûrqa tras su conquista, se cristalizó en una simple purificación al culto cristiano y una adecuación de la mezquita mayor de 1229 levantada sobre un peñasco en el corazón de la ciudad musulmana y junto a la alcazaba de la Almudayna, que fue desapareciendo a medida que la construcción de la Catedral iba avanzando. Su desaparición definitiva se produjo en 1836 (Durliat, 1964; Sureda, 1994; Sastre, 2005).

La nueva construcción recibió el nombre de Catedral de Santa María de Palma de Mallorca. El hijo de Jaime I, el conquistador, Jaime II consolidó la obra (Ripoll, 1945; Durliat, 1964), según las prescripciones de la liturgia y del arte que imprimirían los maestros constructores al templo de singular fisonomía con la construcción de una capilla-mausoleo, bajo la advocación de la Trinidad. Terminadas las obras reales de la Seo, hacia 1330, el Cabildo catedralicio se afanó a adecuar el edificio para el culto, y anotó los gastos en un libro, a cargo de Beniat de Riuclar (1327-1336) y Andreu Porcevl (1337-1345).

La Catedral está situada en el centro de la bahía, imponente sobre su atalaya, y dominado la ciudad y el puerto, superando en altura a todas las demás construcciones. Su silueta era visible desde lejos, tanto desde el mar como desde tierra, afamándose como centro religioso de la ciudad cristiana. A lo largo de las obras, más de 300 años, hubo momentos de euforia constructiva como de letargo. La finalización de la construcción se dio en 1601 con la conclusión de la fachada principal, momento de su consagración por el Obispo Joan Bisbe i Manrique. Su proceso constructivo presenta una serie de particularidades que rompen algunos esquemas tradicionales y difiere de otras construcciones, de similares características, más septentrionales. En 1931, el edificio fue declarado Monumento Histórico-Artístico.

\section{COMPARATIVA PATRIMONIAL ENTRE LAS CATEDRALES DE BARCELONA Y DE MALLORCA}

\subsection{Nombre y Advocación}

Tanto la Catedral de Barcelona como la de Mallorca son llamadas Seu ${ }^{5}$ En su fachada principal o en la lateral importante se abre una plaza que recibe el mismo nombre

La Catedral de Barcelona tiene una doble advocación a la Santa Cruz y a Santa Eulalia. La Catedral de Mallorca está advocada a la Asunción de María

\subsection{Constructores y épocas}

La Catedral gótica de Barcelona inició su construcción en el año 1298, bajo el reinado de Jaime II de Aragón, el justo con el maestro de obras Jaume Fabre al frente. Le siguió Bernat Roca en 1358 hasta 1388 cuando falleció y continuó su picapedrero Pere Viader hasta 1397 encargándose Arnau Bargués y ayudado por Jaume Solá que se ocupó posteriormente. Le sucedió Bartolomé Gual que permaneció largo tiempo en el cargo hasta el año 1441. Nombrado posteriormente Andrés Escuder que dimitiría en el año 1463. La fachada si bien proyectada en el año 1408 por el llamado maestro Carlí no se llevó a cabo hasta finales del siglo XVIII - principios del XIX, por el arquitecto Josep Oriol Mestres que tomó como inspiración los planos del maestro Carlí. El tiempo constructivo de la obra gótica duró 165 años.

La Catedral gótica de Mallorca. Hay indicios documentales, a partir de 1230, relacionados con la obra de la Catedral. En 1269, el obispo Pere de Morella consagró el ara del altar mayor. Pero la construcción tal y como la conocemos se inició en torno al 1300 bajo el reinado de Jaime II, rey de Mallorca (tío de Jaime II rey de Aragón), con el primer maestro de obras Ponç des Coll al que le siguió Jaime Fabre. Según Marcel Durliat, en 1368 los arquitectos eran Jaume Matas y Llorenç Sosquela. Sin embargo, Alexandre Cirici i Pellicer y Agustí Duran i Sampere defienden que el arquitecto al frente del proyecto durante el cambio de plan fue Berenguer de Montagut (Bassegoda Nonell, 1995). Maestro de obras que había trabajado en las Catedrales de Manresa y la Basílica de Santa María del Mar y que conocía perfectamente la estructura de la Catedral de Barcelona. Las obras posteriormente fueron dirigidas entre 1386 y 1397 por Guillem Oliveres. Finalmente, con Guillem Sagrera, arquitecto que también trabajó en Barcelona 1430 se finalizaba la bóveda norte (Alomar Esteve, 1970). Ya en el siglo XX, entre 1904 y 1914, Antonio Gaudí realizó una reforma que duró diez años. El tiempo constructivo de la

\footnotetext{
${ }^{5}$ Según el diccionario de la lengua catalana, se trata del centro de un poder o autoridad especialmente del poder o autoridad del Obispo, También significa Iglesia, Catedral.
} 
obra gótica duró 200 años, si bien hay que considerar que entre 1230 y 1306 es muy poca la actividad constructiva que se dio.

Finalmente, en pleno siglo XX, un artista dejó su huella en ambas Catedrales, Frederic Marés escultor y coleccionista. En el caso de la Catedral de Barcelona, el Cristo Crucificado conocido con el nombre de Exaltación de la Santa Cruz sobre el altar mayor, colocado en el año 1975, y las esculturas en los sepulcros de los reyes Alfonso el Liberal, rey de Aragón y conde de Barcelona y Jaime I de Urgell, conde de Urgell, y Federico de Aragón, hijos de Alfonso el Benigno, en el año 1950. En la Catedral de Mallorca los sepulcros de los Reyes Jaime II y Jaime III situados en su lugar actual, la capilla de la Trinidad, en el año 1947.

\subsection{Materiales constructivos y forma de construir}

Ambas Catedrales utilizaron materiales pétreos del lugar. La cantera propia en el caso de Barcelona fue la de Montjuïc, se trata de un gres de cuarzo del Mioceno compacto y resistente, con coloraciones blancas, ocres e incluso veteadas violáceas (Roca i Blanch, 2003). En el caso de la cantera de Mallorca se cree que parte del material usado, provino de la cala de Portals Vells y que responden a dos de los tipos existentes de piedra carbonatada, exactamente dolomía blanca y caliza blanca (Alonso, Ordaz, \& Esbert, 1996). Ambas canteras aportaban, por tanto, colores claros a las construcciones que se realizaban en sus alrededores.

En ambas Catedrales se optó por derruir lo anterior a medida que avanzaba la nueva construcción, ello les permitió aprovechar la piedra existente en ambas y reaprovechar algunas estructuras, caso de la Catedral de Barcelona, el ara del altar soportada por dos capiteles visigóticos o una puerta románica que conectó más tarde el claustro con la iglesia. Además, como era habitual en las construcciones eclesiásticas ambas Catedrales siguen el principio de empezar a construir por el testero. Pero mientras que en la Catedral de Barcelona las obras no se plantearon como una construcción ex novo sino como una reforma y ampliación de la catedral románica, en la Catedral de Mallorca y debido al hecho que se construía encima de una mezquita, la obra se planteó como una nueva creación.

\subsection{Respecto a la estructura interior y exterior}

Las dos construcciones muestran 3 naves: una principal y dos laterales y con una única puerta de entrada en la fachada principal dividida por un parteluz, y dos puertas laterales, en el caso de la Catedral de Barcelona una de ellas es imperceptible ya que comunica con el claustro y sólo se puede ver desde el interior de la Catedral. La cabecera de la Catedral de Barcelona es de un solo ábside con capillas y deambulatorio, la de la catedral de Mallorca presenta una estructura formada por tres ábsides con capillas y sin deambulatorio.

Figura 1: Planta de las catedrales

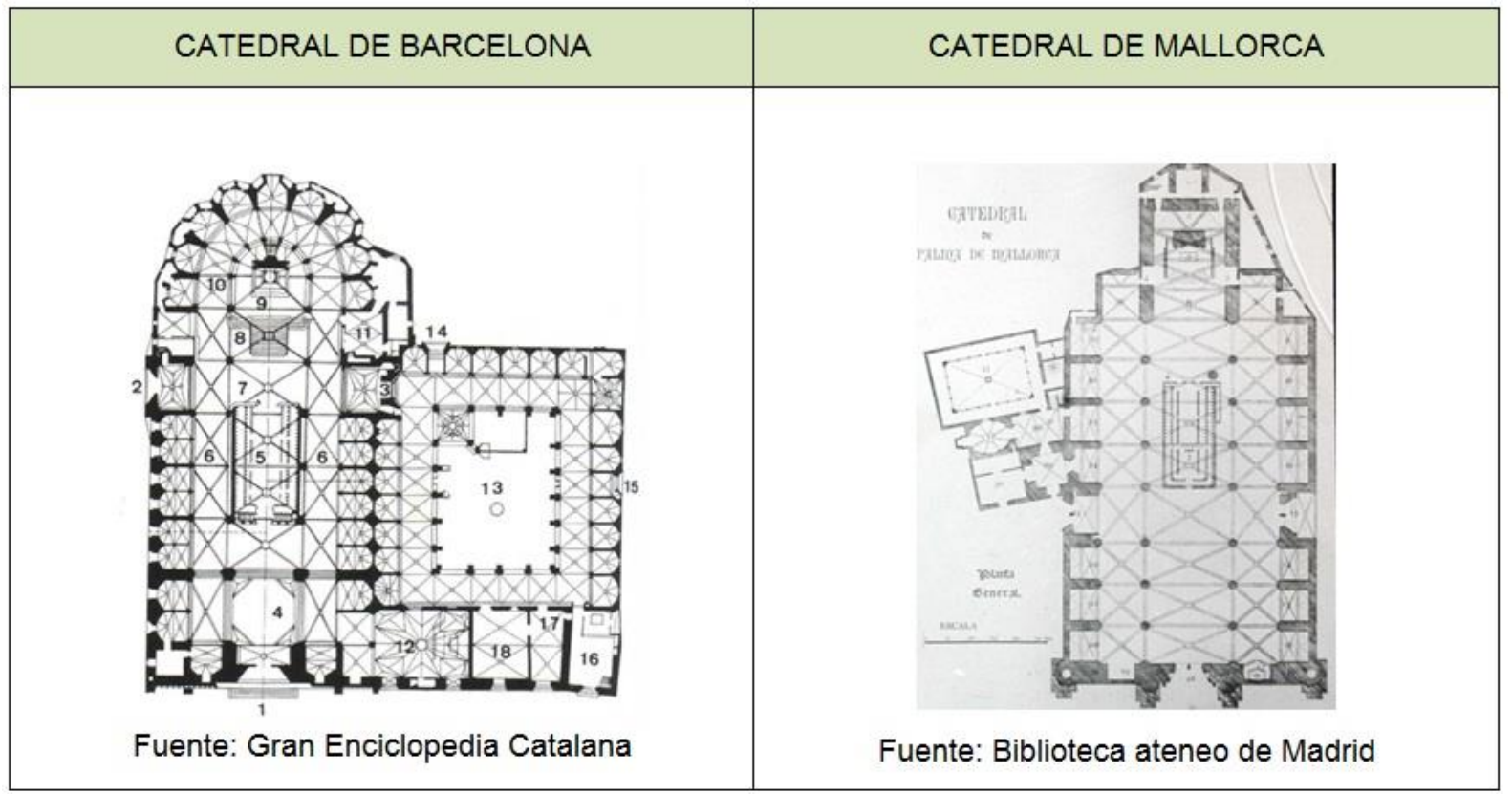

Mientras que la Catedral de Mallorca muestra una planta llamada de salón, la Catedral de Barcelona es una planta de cruz latina, pero visualmente hablando parece de salón, ello es debido a que, en su afán de simplicidad, el gótico de la Corona de Aragón utilizó o la planta salón o la de cruz latina reduciendo al mínimo la importancia del transepto o del crucero que en épocas precedentes había sido de suma importancia. El resultado visualmente acaba siendo el mismo, a nivel de planta, de hecho, cuando llegó al punto álgido de las 
manifestaciones artísticas góticas, se trabajó en aras a la sencillez y desafío arquitectónico con construcciones de planta única y sin naves laterales, como por ejemplo la Catedral de Girona.

Tabla 1: Medidas internas de ambas catedrales

\begin{tabular}{|ll|lc|}
\hline \multicolumn{2}{|c|}{ CATEDRAL DE BARCELONA } & \multicolumn{1}{c|}{ CATEDRAL DE MALLORCA } \\
\hline Longitud nave central & $79 \mathrm{~m}$. & Longitud nave central & $75,52 \mathrm{~m}$. \\
Ancho & $35 \mathrm{~m}$. & Ancho & $39,45 \mathrm{~m}$. \\
Alto nave central & $26 \mathrm{~m}$. & Alto nave central & $43,74 \mathrm{~m}$. \\
Alto naves laterales & $21 \mathrm{~m}$. & Alto naves laterales & $30,17 \mathrm{~m}$. \\
Tramos nave central & 5 & Tramos nave central & 8 \\
Altura de las columnas hasta el & $15 \mathrm{~m}$. & Altura de las columnas hasta el & $21,47 \mathrm{~m}$. \\
arranque de la bóveda & & arranque de la bóveda & \\
\hline
\end{tabular}

Fuente: Elaboración propia basada en las páginas web de ambas catedrales ${ }^{6}$

Tabla 2: Medidas globales de ambas catedrales

\begin{tabular}{|lc|lc|}
\hline \multicolumn{2}{|c|}{ CATEDRAL DE BARCELONA } & \multicolumn{2}{c|}{ CATEDRAL DE MALLORCA } \\
\hline Longitud & $93 \mathrm{~m}$. & Longitud & $109,40 \mathrm{~m}$. \\
Ancho & $40 \mathrm{~m}$. & Ancho & $39,45 \mathrm{~m}$. \\
Alto nave central & $28 \mathrm{~m}$. & Alto nave central & $43,95 \mathrm{~m}$. \\
Alto naves laterales & $23 \mathrm{~m}$. & Alto naves laterales & $29,49 \mathrm{~m}$. \\
\hline
\end{tabular}

Fuente: Elaboración propia basada en las páginas web de ambas catedrales

En cuanto a longitud y anchura de las naves centrales y laterales a nivel interno las medidas son muy parecidas. Mostrando al visitante un criterio de compactación y sobriedad típico del gótico catalán de la Corona aragonesa.

Pero en cuanto a altura interior los datos nos muestran una variable importante ya que conduce a una determinada construcción. Mientras la Catedral de Barcelona entre la nave central y las naves laterales presenta un $23,80 \%$ de diferencia altimétrica, la Catedral de Mallorca es de un 44,97\%. Esta diferencia es mucho más perceptible a nivel exterior que interior, puesto que mientras ese $33,33 \%$ de la Catedral de Barcelona evita la construcción de nuevos contrafuertes, la de Mallorca con el 44,97\% provoca justamente la necesidad de construir otros contrafuertes, los llamados arbotantes, tan trabajados en el gótico francés y en el gótico catalán de la Corona de Aragón, cercano a Francia, como es la Catedral de Girona que lo hizo, exclusivamente, en la zona del ábside. (Catedral de Barcelona, 2013), (Catedral de Mallorca, 2014)

Los contrafuertes interiores. Típico del gótico de la Corona de Aragón fue el reaprovechamiento de espacios. Los muros laterales internos mostraban los contrafuertes que soportaban la estructura de las naves laterales y éstas a su vez de la nave central. Pero tanto en la Catedral de Barcelona como en la de Mallorca reaprovecharon los vacíos que quedaban entre los contrafuertes para situar las capillas. Llenar esos vacíos permitía visualmente ofrecer una imagen uniforme, donde los contrafuertes quedaban absorbidos por las capillas y englobados hasta la cubierta de las capillas por un único muro perimetral. A nivel exterior la percepción del visitante es sólo una, un lienzo plano que resigue la Catedral.

Ambas Catedrales asumieron y construyeron en base a este concepto. Pero hubo una razón también financiera para seguir con esta forma constructiva. La búsqueda de financiación ser resolvió alquilando o vendiendo dichas capillas siendo sus clientes más directos los gremios y las cofradías que adquirían una serie de derechos a cambio de ese alquiler o venta (reunirse en la capilla, celebración de misas exclusivamente gremiales, etc.). En el caso de Barcelona, fueron las Cofradías y los Gremios medievales y en el caso de la Catedral de Mallorca fueron la Cofradía de Nuestra Señora Santa María, las donaciones de algunos Gremios y el llamado pago de la Anata Eclesiástica?.

Tabla 3: Las Capillas

\begin{tabular}{|lc|lc|}
\hline CATEDRAL DE BARCELONA & & CATEDRAL DE MALLORCA & \\
\hline Alto capillas laterales & $9 \mathrm{~m}$. & Alto capillas laterales & $16,35 \mathrm{~m}$. \\
Ancho capillas laterales & $5 \mathrm{~m}$. & Ancho capillas laterales & $7,65 \mathrm{~m}$. \\
Profundidad capillas laterales & $6 \mathrm{~m}$. & Profundidad capillas laterales & $6,70 \mathrm{~m}$. \\
Número de capillas $^{8}$ & 27 & Número de capillas & 16 \\
\hline
\end{tabular}

Fuente: Elaboración propia basada en las páginas web de ambas catedrales

\footnotetext{
${ }^{6}$ http://www.catedralbcn.org y http://www.catedraldemallorca.info (Páginas consultadas durante mes de febrero de 2014)

${ }^{7}$ Según el Diccionario de la Real Academia de la Lengua española se trataba de un impuesto eclesiástico que consistía en la renta o frutos correspondientes al primer año de posesión de cualquier beneficio o empleo.

${ }^{8}$ Sólo se consideran las del interior de la iglesia, no las del claustro.
} 
En su origen las dos Catedrales situaron en el centro de la nave Central el coro. La Catedral de Barcelona aún lo mantiene en el mismo sitio, mientras que la Catedral de Mallorca lo traslado al altar Mayor o Capilla Real.

Finalmente, en cuanto a estructuras las dos presentan un claustro, aunque en ubicaciones distintas debido básicamente al hecho de reaprovechamiento de estructuras precedentes. Hay que tener presente que en una Catedral no es necesaria la construcción de claustro, véanse casos como Nôtre Dame de Paris, Duomo de Milán, - la Catedral de Colonia). Si se construía un claustro era porque existía una Canóniga9 ${ }^{9}$ (Vila i Martin \& Puig Closa, 2008).

\subsection{Tumbas Reales}

No necesariamente una Catedral se convertía en un panteón para los reyes, pero en el caso de las Catedrales que aquí nos ocupan, ambas fueron utilizadas como mausoleos para los altos cargos dignatarios más importantes de la época, los reyes, así como de los Obispos más destacados. En el caso de la Catedral de Mallorca, y por ser Jaume II el primer rey del reino de Mallorca no había otras construcciones eclesiásticas que sirvieran de mausoleo real. Pero en el caso de la Catedral de Barcelona, su antecesora románica, ya había servido de mausoleo a las más altas dignidades de la época precedente, los Condes de Barcelona.

Tabla 4: Reyes Enterrados

\begin{tabular}{|l|c|}
\hline \multicolumn{1}{|c|}{ CATEDRAL DE BARCELONA } & CATEDRAL DE MALLORCA \\
\hline El rey Alfonso el Liberal o Franco. & $\square$ Jaime II, rey de Mallorca \\
Jaime I de Urgell, conde de Urgell, hijo del rey Alfonso el & $\square$ Jaime III, nieto de Jaime II \\
Benigno & \\
Federico de Aragón, hijo del rey Alfonso el Benigno & \\
Constanza de Sicilia, esposa de Pedro el Grande & \\
María de Chipre, esposa de Jaime el Justo & \\
Sibila de Fortiá, esposa de Pedro el Ceremonioso \\
Leonor de Aragón y Foix, hija de Pedro IV de Ribagorza y \\
segunda esposa de Pedro I de Chipre, rey de Chipre y \\
Jerusalén.
\end{tabular}

Fuente: Elaboración propia basada en las páginas web de ambas catedrales

\section{COMPARATIVA TURÍSTICA ENTRE LAS CATEDRALES DE BARCELONA Y DE MALLORCA}

Este estudio comparativo se hizo durante el mes de marzo de 2014 y no hubiera sido posible hacerlo sin la amabilidad y la ayuda del gerente y del personal del Cabildo Catedralicio de la Catedral de Palma de Mallorca, guías y personal adscrito a ambas catedrales. Se formuló un cuestionario que recibió respuesta por parte del Cabildo Catedralicio de la Catedral de Mallorca, en cambio para obtener los datos de la Catedral de Barcelona se tuvo que acudir a fuentes indirectas, como preguntas contrastadas in situ por guías (total de 5: 3 mujeres y 2 hombres) y personal de los servicios en el interior del recinto (1 mujer que trabajaba en la tienda de la Catedral). También agradecemos la labor desinteresada por parte del personal de los servicios bibliotecarios de las distintas facultades de la Universidad de les Illes Balears y de la Biblioteca Cultural Artesana de Palma, que se han volcado en la búsqueda de los textos bibliográficos.

Se partía del hecho de considerar a ambas Catedrales recurso turístico. Hecho confirmado tanto por el cuestionario como por los datos logrados in situ. Además, en las propias páginas web de ambos recursos patrimoniales, dedican dos epígrafes: 1. Tipos de actividades turísticas.2. Horarios y precios para los visitantes.

Respecto a actividades obtuvimos los siguientes datos: El abanico de actividades se mostraba pensando no sólo en una actividad comercial como tener una tienda de recuerdos, sino por ejemplo en las visitas turísticas a los distintos espacios, o si se realizaban talleres y/o actividades de conferencias y seminarios.

\footnotetext{
${ }^{9}$ Canóniga, comunidad de clérigos que se rigen por una regla o canon y son llamados canónigos o clérigos regulares (sacerdotes). Su carácter era secular y no regular, lo que marcaba su carácter abierto.
} 
Tabla 5: Tipos de Actividades

\begin{tabular}{|l|l|}
\hline Visitas guiadas del recinto Catedralicio a los turistas & $20 \%$ \\
\hline Visitas guiadas del recinto Catedralicio a las escuelas (pedagógicas) & $20 \%$ \\
\hline Visitas por propia iniciativa del turista & $20 \%$ \\
\hline Tienda de Recuerdos & $20 \%$ \\
\hline Museo Catedralicio & $20 \%$ \\
\hline Conferencies y/o Seminarios & $10 \%$ \\
\hline Otras & $0 \%$ \\
\hline
\end{tabular}

Fuente: Elaboración propia en base al cuestionario y datos aportados desde las páginas web

Como se puede apreciar en base a las respuestas (ver tabla 6.1) había un único valor divergente, la variable de conferencias y/o seminarios sólo la Catedral de Palma realiza dicha actividad. Respecto a los horarios y precios (ver tabla 6 y 7) pudimos observar una variedad tanto en el primer elemento como en el segundo. De modo que se permitía la entrada a los turistas todos los días en las horas que la Catedral tiene abierta.

Tabla 6: Horarios, Visitas Turísticas y Precios

\begin{tabular}{|c|c|c|c|c|c|}
\hline \multicolumn{2}{|l|}{ Horarios Visitas Turísticas } & \multicolumn{4}{|c|}{ Precios } \\
\hline Durante todo el día & $100 \%$ & & & & \\
\hline Sólo por las mañanas & $0 \%$ & \multirow{2}{*}{\multicolumn{2}{|c|}{$\begin{array}{l}\text { CATEDRAL DE } \\
\text { BARCELONA }\end{array}$}} & \multirow{2}{*}{\multicolumn{2}{|c|}{$\begin{array}{l}\text { CATEDRAL DE } \\
\text { MALLORCA }\end{array}$}} \\
\hline Sólo por las tardes & $0 \%$ & & & & \\
\hline Sólo al mediodía & $0 \%$ & \multirow{2}{*}{\multicolumn{2}{|c|}{$\begin{array}{l}\text { Precios variables en } \\
\text { función de lo que se } \\
\text { visita }\end{array}$}} & \multirow{2}{*}{\multicolumn{2}{|c|}{ 口 Precio único }} \\
\hline Otros & $0 \%$ & & & & \\
\hline
\end{tabular}

Fuente: Elaboración propia en base al cuestionario y datos aportados desde las páginas web

Y finalmente nos interesaba saber qué espacios eran visitables. (Ver tabla 7) Los espacios coincidentes son el interior de la iglesia. Mientras la Catedral de Barcelona añade las capillas la Catedral de Palma no, en ambas se visitan el claustro y el museo. Destaca el aprovechamiento que hace la Catedral de Barcelona con sus terrazas y la subida a ellas por la torre campanario (ascensor) mientras que la Catedral de Palma es un espacio que, hoy por hoy, no está destinado a visita turística.

Tabla 7: Tabla y Gráfico circular sobre los Espacios Visitables

\begin{tabular}{|c|c|}
\hline \multicolumn{2}{|l|}{ Espacios Visitables } \\
\hline $\begin{array}{l}\text { CATEDRAL DE } \\
\text { BARCELONA }\end{array}$ & $\begin{array}{l}\text { CATEDRAL DE } \\
\text { MALLORCA }\end{array}$ \\
\hline \begin{tabular}{|ll} 
& $\begin{array}{l}\text { Interior de la } \\
\text { Iglesia con }\end{array}$ \\
capillas \\
& La Cripta \\
& El Claustro \\
& Las terrazas \\
& La Torre \\
Campanario \\
El Museo
\end{tabular} & $\begin{array}{ll}\square & \text { Interior iglesia sin } \\
& \text { capillas } \\
\square & \text { Claustro } \\
\square & \text { Museo }\end{array}$ \\
\hline
\end{tabular}

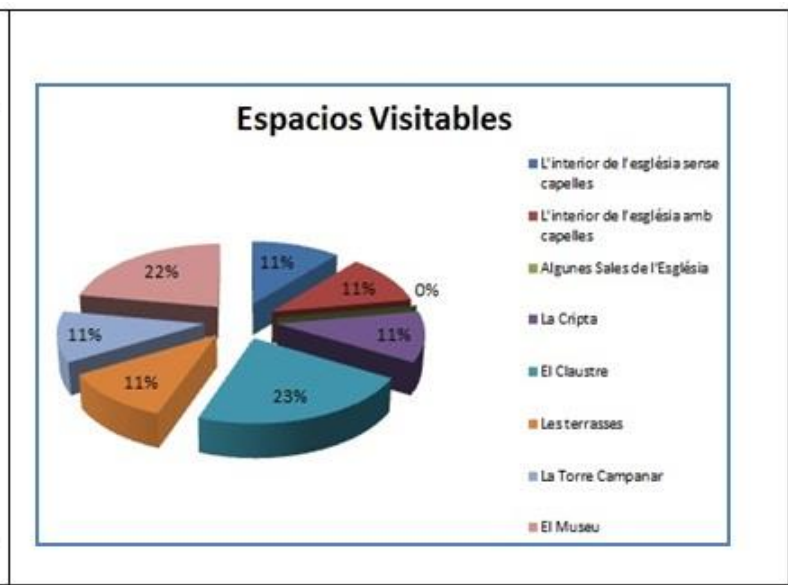

Fuente: Elaboración propia en base al cuestionario y datos aportados desde las páginas web

Siguiendo la clasificación que realiza CICATUR-OEA, se trata de un recurso de jerarquía 2, es decir atractivo con rasgos excepcionales, capaz de motivar una corriente de visitantes. En definitiva, no son sólo un recurso cultural turístico por el aporte histórico arquitectónico que aportan sino porque parte de su gestión lleva a entenderlo como tal. 


\section{CUADRO RESUMEN Y CONCLUSIONES}

Tabla 8: Cuadro Resumen

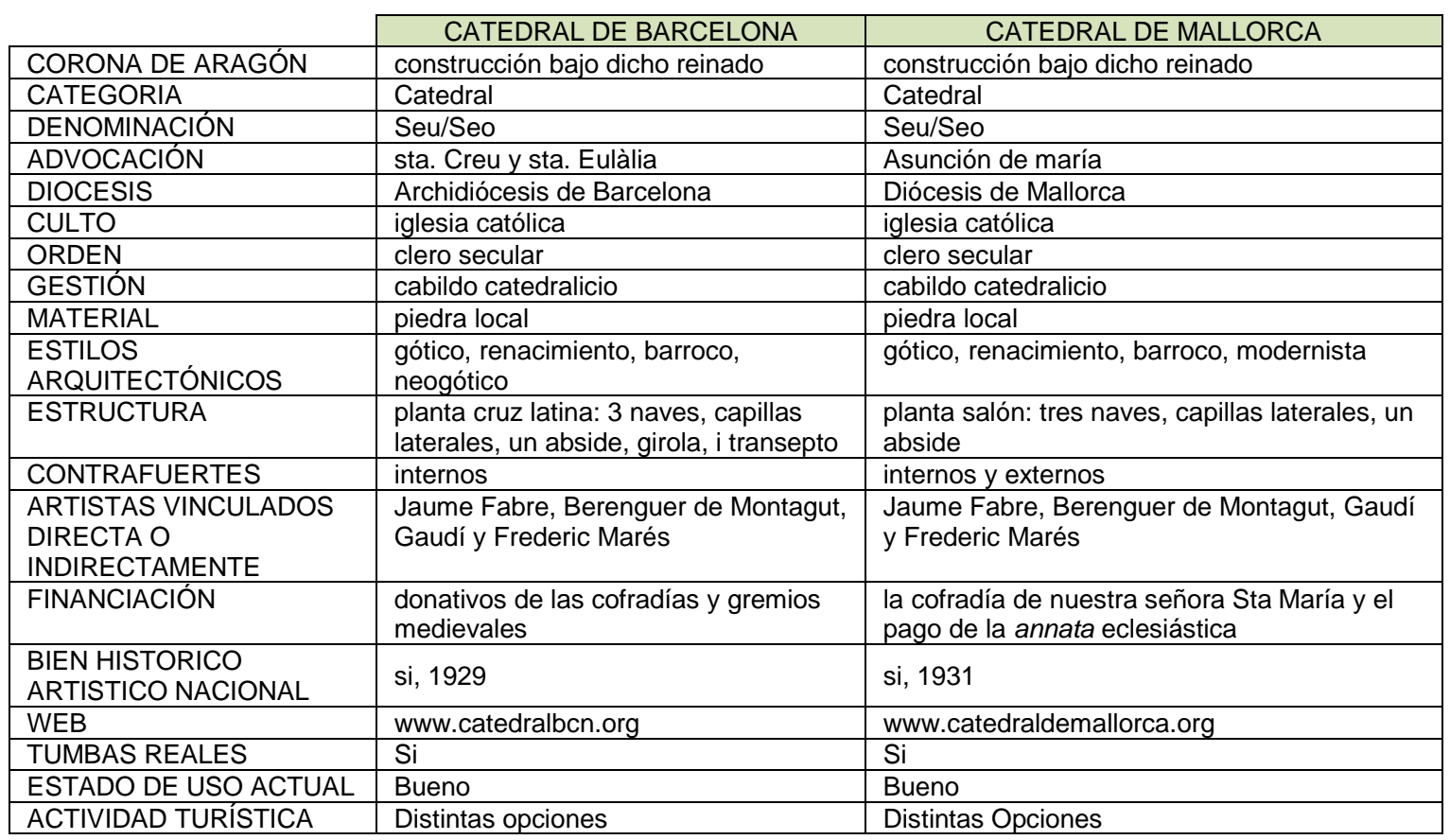

Fuente: Elaboración propia

En base a la información aportada podemos afirmar que hay una serie de elementos comunes a ambas Catedrales que van desde: el estilo gótico, con peculiaridades propias de cada territorio, pasando por el renacentista y el barroco básicamente en elementos decorativos que ambas fueron construidas con la piedra local; que un maestro de obras en común fue Jaume Fabre y un Escultor Frederic Marés, aunque ambas se vieron entrelazadas por artistas de otros tiempos, caso de Berenguer de Montagut o Gaudí y finalmente ambas son sede de panteones reales, están declaradas Bien Histórico Artístico Nacional.

En cuanto a la gestión turística difieren bastante en la manera de llevarla a cabo, si bien ambas permiten las visitas turísticas (dejamos a un lado las visitas pedagógicas), la realidad es que tienen poco desarrollado dicho concepto. La de Mallorca deja la gestión de las visitas guiadas turísticas a los guías de la ciudad mientras que la de Barcelona deja también la gestión a los guías locales pero apuesta por un cuerpo de voluntariado.

Aunque los espacios a visitar son distintos, hay tres áreas que forman la columna vertebral de las visitas el interior, el claustro y el Museo, ofreciendo la Catedral de Barcelona espacios como las terrazas, las capillas y la torre campanario a modo de diferencia sustancial. Sus horarios son dispares al igual que la política de precios, siendo mucho más uniforme la gestión de la Catedral de Mallorca que la de Barcelona, hecho que beneficia la labor de los guías y el disfrute del recurso por parte de los turistas

Finalmente creemos que como recurso turístico - religioso ambas Catedrales tienen que ganar notoriedad, ser un recurso de jerarquía 2 en la escala de CICATUR no es desdeñable, pero si mejora su gestión e incluso llegan a plantearse algún producto en común, tipo pasaporte a las Catedrales bajo la Corona de Aragón, su posicionamiento en el ámbito turístico puede que se llegue a consolidar y a formar parte indisoluble de los atractivos de jerarquía 1 que poseen ambas ciudades.

\section{REFERENCIAS}

Ainaud de Lasarte, J. (1992). La arquitectura gótica religiosa. (29), 20-25.

Alomar Esteve, G. (1970). Guillem de Sagrera y la arquitectura gótica del siglo XV. Barcelona: Blume.

Alonso, F., Ordaz, J., \& Esbert, R. (1996). Deterioro selectivo de la piedra de construcción de la Catedral de Palma de Mallorca. Geogaceta, 1228-1231.

Bassegoda Nonell, J. (1995). Els treballs i les hores a la Catedral de Barcelona. Barcelona: Reial Academia Catalana de Belles Arts de Sant Jordi.

Blasco Peris, A. (2006). Tipologías turísticas. Girona: Xarxa d'Escoles de Turisme. 
Catedral de Barcelona. (2013). catedralbcn. Recuperado en el mes de febrero de 2014, de catedralbcn: http://www.catedralbcn.org/

Catedral de Mallorca. (2014). catedraldemallorca. Recuperado en el mes de febreo de 2014, de catedraldemallorca: http://www.catedraldemallorca.info/

Fàbrega i Grau, A. (1968). La Catedral de Barcelona. Guía Turística. Sèrie III. Monografies. . Barcelona: Arxiu Capitular de la Catedral de Barcelona.

García Martos, M. (2012). Emmanuel: El Oro de los Dioses (Vol. 1). España.

Le Goff, J.; Schmitt, J.C. (2003). Diccionario razonado del Occidente medieval. Madrid: Akal.

Marrero Rodríguez, J. (2009). Las bases del turismo moderno y el desarrollodel turismo de masas. En J. Marrero Rodríguez, \& M. González Ramallal, Manual de Sociología del Ocio Turístico (págs. 143-165). Oviedo: Septem ediciones.

Montaner Montejano, J. (1991). Estructura del Mercado Turístico. Barcelona: Síntesis.

Organización Mundial de Turismo. (1995). Concepts, definitions and classifications for tourism statistics. Madrid: Organización Mundial de Turismo.

Pulido Fernández, J.I.; Sáez Cala, Antonia. (2011). Estructura general del mercado turístico. Madrid: Síntesis.

Roca i Blanch, E. (2003). Barcelona, metròpolis mediterrania. Recuperado el 14 de 04 de 2014, de Barcelona, filla de Montjuic: http://www.bcn.cat/publicacions/b_mm/bmm61/bmm61_qc38.htm

Vila i Martin, M., \& Puig Closa, A. (2008). Gremis i làpides al claustre de la Catedral de Barcelona. Barcelona: Catedral de Barcelona. 